\title{
Predicting outcomes and drug resistance with standardised treatment of active
} tuberculosis

\author{
O. Oxlade ${ }^{\star, \#}$, K. Schwartzman*,\#, M. Pai*,\#, J. Heymann", A. Benedetti*,\#, \\ S. Royce ${ }^{+}$and D. Menzies*
}

ABSTRACT: New World Health Organization guidelines recommend initial treatment of active tuberculosis (TB) with a 6-month regimen utilising rifampin throughout. We have modelled expected treatment outcomes, including drug resistance, with this regimen, compared to an 8month regimen with rifampin for the first 2 months only, followed by standardised retreatment.

A deterministic model was used to predict treatment outcomes in hypothetical cohorts of 1,000 new smear-positive cases from seven countries with varying prevalence of initial drug resistance. Model inputs were taken from published systematic reviews. Predicted outcomes included number of deaths, failures and relapses, plus the proportion with drug resistance. Sensitivity analyses examined different risks of acquired drug resistance.

Compared to use of the standardised 8-month regimen, for every 1,000 new TB cases treated with the 6-month regimen we predict that 48-86 fewer persons will require retreatment, and 3-12 deaths would be avoided. However, the proportion failing or relapsing after retreatment is predicted to be higher, because with the 6-month regimen $50-94 \%$ of failures and $3-56 \%$ of relapses will have multidrug-resistant TB.

We predict substantial public health benefits from changing from the 8-month to the 6-month regimen. However in almost all settings the current standardised retreatment regimen will no longer be adequate.

KEYWORDS: Acquired drug resistance, modelling, relapse, treatment failure, tuberculosis treatment

ince the early 1990s the World Health Organization (WHO) [1] and the International Union Against TB and Lung Disease [2] have recommended use of a limited number of standardised drug regimens to treat active tuberculosis (TB) cases. This approach ensures that patients receive appropriate drugs in the right doses, for the correct length of time, and has been adopted in most low- and middleincome countries [3]. Up to now two regimens have been recommended for new patients. The "8-month regimen" includes isoniazid, rifampin, pyrazinamide and ethambutol for 2 months followed by isoniazid plus ethambutol for 6 months (2HRZE/6HE). The "6-month regimen" includes isoniazid, rifampin, pyrazinamide and ethambutol for 2 months, followed by isoniazid plus rifampin for 4 months (2HRZE/4HR). The 6-month regimen has higher efficacy [4], but the 8-month initial regimen is preferred in countries where resource limitations preclude supervision of rifampin in the continuation phase [2]. In view of the greater efficacy, the WHO has recently recommended that the 6-month regimen be used as standardised initial therapy in all countries [5].

For all previously treated patients who have failed, relapsed or returned after failing to complete (defaulting) initial treatment, the WHO had recommended a single standardised retreatment regimen [1]. This consisted of 2 months of streptomycin, isoniazid, rifampin, pyrazinamide and ethambutol, followed by 1 month of isoniazid, rifampin, pyrazinamide and ethambutol, followed by 5 months of isoniazid, rifampin and ethambutol (2SHRZE/ 1HRZE/5HRE). WHO estimates that $\sim 12 \%$ of all currently treated patients receive retreatment,

\section{AFFILIATIONS}

*Respiratory Epidemiology and Clinical Research Unit, Montreal Chest Institute, McGill University, \# Dept of Epidemiology, Biostatistics and Occupational Health, McGill University, and

-Institute of Health and Social Policy, McGill University, Montreal, QC,

Canada.

+University of California, San

Francisco, CA, USA.

\section{CORRESPONDENCE}

D. Menzies

McGill University

Respiratory Epidemiology Unit

Montreal Chest Institute

3650 St Urbain

Room K1.24

Montreal

QC

H2X 2P4

Canada

E-mail: dick.menzies@mcgill.ca

Received:

Sept 252009

Accepted after revision:

March 132010

First published online:

March 312010 
meaning as many as 1.1 million individuals receive this regimen, given the total annual incidence of 9.2 million cases [3].

This standardised retreatment regimen was never tested in randomised trials [6], but rather was designed for use in subSaharan Africa for patients who had initially received the 8month regimen and had very low likelihood of multidrugresistant (MDR) [7]. Use of the same retreatment regimen following the 6-month initial treatment has been particularly controversial $[8,9]$, because treatment outcomes are poor in settings with high prevalence of initial drug resistance [10], and use of this regimen is associated with amplification of drug resistance [11, 12]. Surveillance information has consistently shown that the prevalence of drug resistance is higher among previously treated cases than it is among new cases [13]. However, there is very limited and contradictory surveillance data linking drug resistance to detailed clinical histories, such as whether patients had previously defaulted, failed or relapsed after apparent cure [11, 12, 14, 15]. If the prevalence of drug resistance is very high in any of the retreatment subgroups (failure, relapse or prior default) following the initial 6-month regimen, it would be inappropriate to treat them empirically with the current retreatment regimen.

This modelling exercise was undertaken in order to inform the treatment revision guidelines published by the WHO. These new guidelines have recently been made available, and now recommend that, in settings with no access to drug sensitivity testing, failures of initial treatment should be offered an empirical MDR regimen [5], pending DST results. Those who have relapsed and defaulted, however, will continue to be offered the standardised retreatment regimen, pending DST results, unless country specific data show high levels of MDR in such patients.

We have used modelling to predict the treatment outcomes of failure and relapse, as well as the pattern of drug resistance associated with each of these outcomes, following initial therapy with one of two standardised initial regimens and the current standardised retreatment regimen, in settings with varying levels of drug resistance.

\section{METHODS}

\section{Overview of model}

A deterministic model representing a decision tree was developed to simulate hypothetical cohorts of 1,000 smearpositive active TB cases undergoing a single round of initial treatment and retreatment, all of which occurred within a year. New cases received either the standardised 8- or 6-month regimen; those who failed or relapsed received the standardised 8-month retreatment regimen. A simplified outline of the model is provided in the online supplementary material. Cohorts were modelled in seven countries, selected to represent widely varying prevalence of initial drug resistance. The probability of transitioning at each decision node was determined from data found in the literature. Within each country the probability of cohort members starting with underlying drug resistance was determined by the drug resistance prevalence in that country from WHO reports [13]. The probabilities of failure, relapse and acquired drug resistance were based on the regimen received (6- or 8-month) and the underlying drug resistance; these probabilities were taken from results of two recent systematic reviews and metaanalyses of randomised trials [4, 6]. Model predicted outcomes included deaths, the numbers who relapsed or failed initial therapy, began retreatment then failed or relapsed, and the proportion with drug resistance among failures or relapses. The total number of outcomes that occurred with each strategy was summed and compared using basic spreadsheet analysis. Models were validated using published drug resistance data from countries that used either the 6- or 8-month regimens for new cases. Uncertainty in key parameters was addressed in sensitivity analysis (see section below).

\section{HIV}

In a recent meta-analysis there were very few randomised trials or cohort studies of treatment of HIV co-infected patients with underlying drug resistance, and too few patients with drug resistance for pooled estimates of outcomes [16]. Therefore, we assumed that model predicted outcomes would be similar for TB cases with HIV and without HIV. There is some evidence that the acquisition of drug resistance may be increased in TB cases that are HIV positive [17]. We explored this possibility in a sensitivity analysis in which we varied the risk of acquisition of drug resistance.

\section{Initial drug resistance profiles}

Prevalence of initial drug resistance in each country (table S1 in the online supplementary material) was taken from the most recent WHO surveillance report on drug resistance [13]. Initial drug resistance was categorised as: pan-susceptible, monoisoniazid resistant, mono-streptomycin resistant, mono-ethambutol resistant, mono-rifampin resistant, poly-drug resistant (PDR) (defined as resistant to two or more drugs, but not meeting the definition of MDR) and MDR (as defined above). Mono-rifampin resistant cases were grouped with MDR cases. The mono-streptomycin resistance group was considered equivalent to the pan-susceptible group because streptomycin is not included in standardised initial treatment, except in Vietnam, where streptomycin is used, so this form of resistance was modelled with distinct treatment outcomes $[4,18]$.

\section{Modelling treatment}

In $\mathrm{TB}$ programmes in low- and middle-income countries, the pre-treatment drug resistance is not known to practitioners; all treatment is standardised and empirical. Therefore, we assumed that standardised initial and retreatment regimens were given to all patients, regardless of underlying drug resistance profiles.

As shown in the supplementary figure, with initial treatment, new cases could be cured, or die or fail during initial treatment. The proportion of the hypothetical cohort that died during initial treatment was $5.6 \%$ for all cases with either initial regimen (based upon global reported mortality during initial treatment in 2003 and 2004 for patients taking standardised initial treatment [10]), except for MDR cases, as described below. A proportion of the cohort could fail, and the remainder were cured, of whom some could relapse. We assumed that all patients would complete treatment; the impact of noncompletion (defaulters) was addressed in sensitivity analyses. Failure and relapse rates varied according to the standardised initial treatment regimen received (8- or 6-month), and underlying drug resistance (see section below). Outcomes for MDR 
cases after initial standardised treatment were $25 \%$ spontaneous cure and $33 \%$ mortality. In the absence of other data, we assumed that TB cases with MDR have the same mortality and spontaneous cure rate as untreated cases in Europe in the preantibiotic era [19]). The remainder of MDR cases failed (42\%).

We assumed that all failures and relapses would be detected and receive the standardised retreatment regimen. Those who required retreatment were reclassified according to their predicted post-treatment drug resistance profiles. Outcomes were modelled in a similar manner as for initial treatment, except that spontaneous cure of MDR-TB would not occur a second time (since we assumed the maximum reported value for spontaneous cure would occur during initial treatment). All retreatment cases could be cured, die or fail during retreatment or relapse after cure, with probabilities determined by their pre-treatment drug resistance, but independent of whether they required retreatment because of failure or relapse with initial treatment. Mortality during retreatment for all nonMDR cases was 7.8\% (based upon global reported retreatment mortality in 2003 and 2004 for patients taking standardised retreatment [10]), and was 33\% for MDR cases [19].

\section{Failure/relapse and acquired resistance rates}

Treatment failure, relapse and acquired drug resistance rates for the initial 6- and 8-month regimens, according to underlying initial drug resistance as defined above, were taken from a systematic review and meta-analysis of 57 published randomised trials [4]. In order to address the problem of increased risk of acquired drug resistance associated with suboptimal adherence in true programme settings a sensitivity analysis was conducted (see below). There are no randomised trials reporting outcomes of the currently recommended standardised retreatment regimen [6]. There are only seven cohort studies that report bacteriologically confirmed outcomes in individual patients receiving the retreatment regimen; of these, only three reported outcomes in isoniazid resistant cases and none reported outcomes with other forms of drug resistance [6]. Hence, probabilities of failure, relapse and acquired drug resistance for the standardised retreatment regimen were assumed to be the same as the probabilities for the 6-month initial regimen. This assumption was investigated in sensitivity analysis (see below).

\section{Outcomes estimated}

Using all of the above data, the total number of failures, relapses and deaths, plus the resistance profile among those who relapsed or failed, were predicted following initial treatment with the 6- or 8-month regimen and standardised retreatment.

\section{Validation}

We applied published data on the prevalence of initial drug resistance [13], in order to compare prevalence predicted using our model with observed prevalence of MDR among failures and relapses in Peru [14] and Thailand [12], where the 6-month regimen was used, and Benin [15], where the 8-month regimen was used.

\section{Sensitivity analysis}

Three sensitivity analyses were conducted. The first investigated the potential influence of defaulting by increasing the relapse rates with initial and retreatment regimens by $20 \%$, well above the global average default rate [3]. The second increased the probability of acquiring drug resistance during initial treatment by $25 \%$. This sensitivity analysis provided insight into the possibility that the risk of acquired drug resistance would be higher in true programme settings, or increased in HIV co-infected patients. The third sensitivity analysis increased the efficacy of the retreatment regimen by decreasing failure, relapse and acquired drug resistance rates by $25 \%$.

\section{RESULTS}

\section{Predicted failures, relapses, and patterns of drug resistance among failures and relapses}

As shown in table 1 the predicted number of failures of initial therapy was most strongly affected by the prevalence of initial drug resistance, although failures were more frequent with the 8-month regimen in all settings. However, the proportion with MDR would be much higher following initial treatment with the 6-month regimen. In almost all countries, virtually all failures of standardised retreatment were predicted to have MDR, regardless of initial drug resistance or initial regimen.

Because of much higher relapse rates, the predicted total number of patients requiring retreatment will be much higher following the 8-month regimen, particularly with higher prevalence of initial drug resistance as shown in figure 1. The proportion with MDR, or any form of drug resistance, would be much lower among relapses than among failures with initial therapy, but much higher after standardised retreatment, as seen in table 2.

As summarised in table 3, the majority of drug resistance after initial and retreatment was persistent, meaning that it had been present even before treatment, and simply persisted unchanged throughout therapy. However, some acquired drug resistance did occur; with the 8-month regimen this occurred more frequently but was usually mono-resistance; however with the 6-month regimen acquired drug resistance was less frequent, but more serious, as it was usually MDR.

The difference in drug resistance patterns was much greater between failures and relapses, than they were between the different initial regimens, as demonstrated in figures 2 and 3.

\section{Validation}

The predictions from this modelling study compare well with published surveillance data in a few countries. Among countries using the 6-month initial regimen (fig. 2), in Peru, the prevalence of MDR among failures of the 6-month regimen was 94\% [14], close to our predicted prevalence of $87 \%$. In Thailand, the prevalence of MDR was reported to be $86 \%$ in failures and $11 \%$ in relapses [12], compared to predicted values of $80 \%$ in failures and $21 \%$ in relapses. In Benin, where the 8 -month initial regimen was used, (fig. 3), our model predicted that $14 \%$ of failures and $1 \%$ of relapses would have MDR, compared to a reported prevalence of $22 \%$ and $4 \%$, respectively [15].

\section{Comparison of outcomes including deaths, with the two initial treatment approaches}

As seen in table 4, deaths were most strongly associated with prevalence of drug-resistant TB. In countries with high rates of initial drug resistance, a substantial proportion of deaths were 


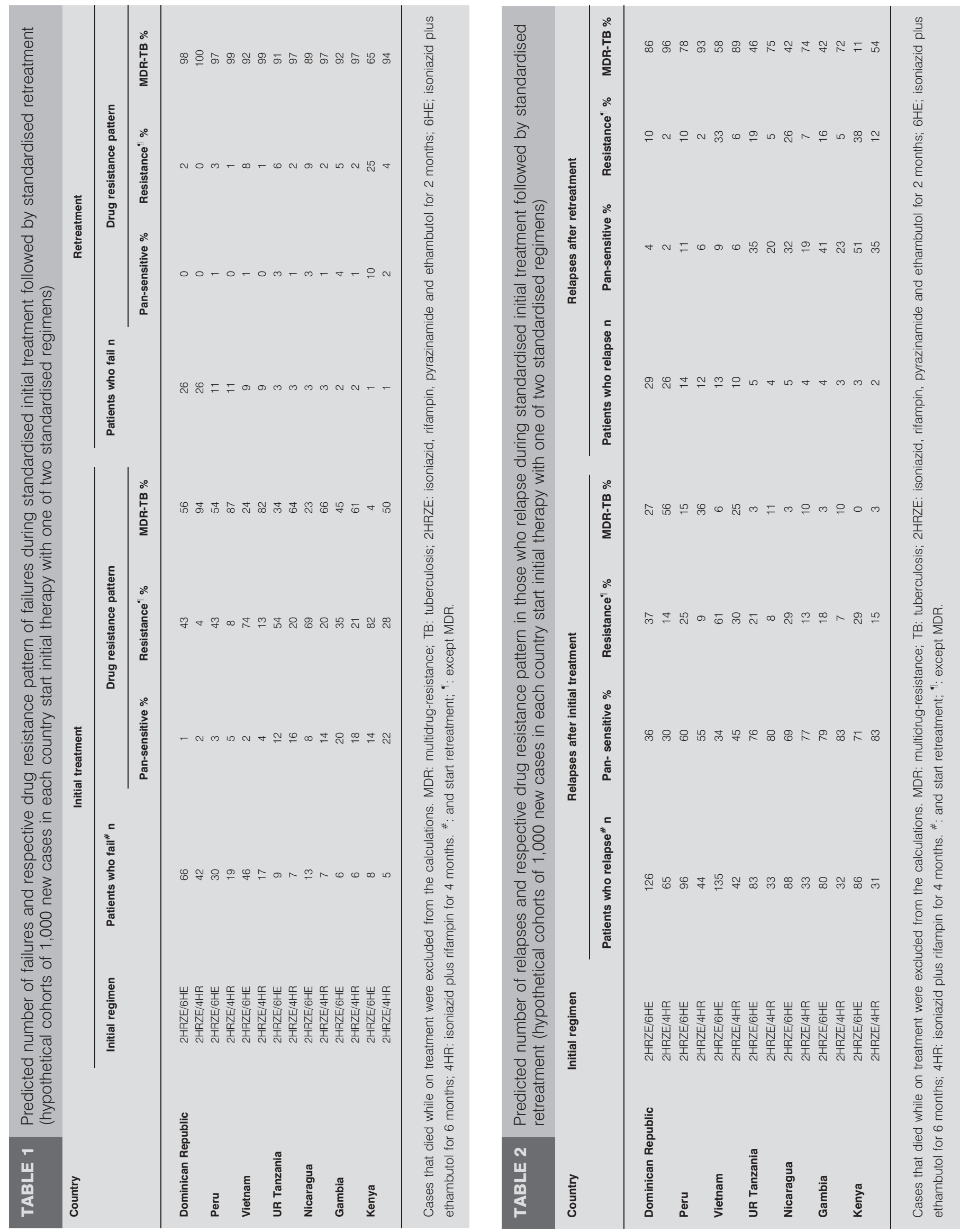




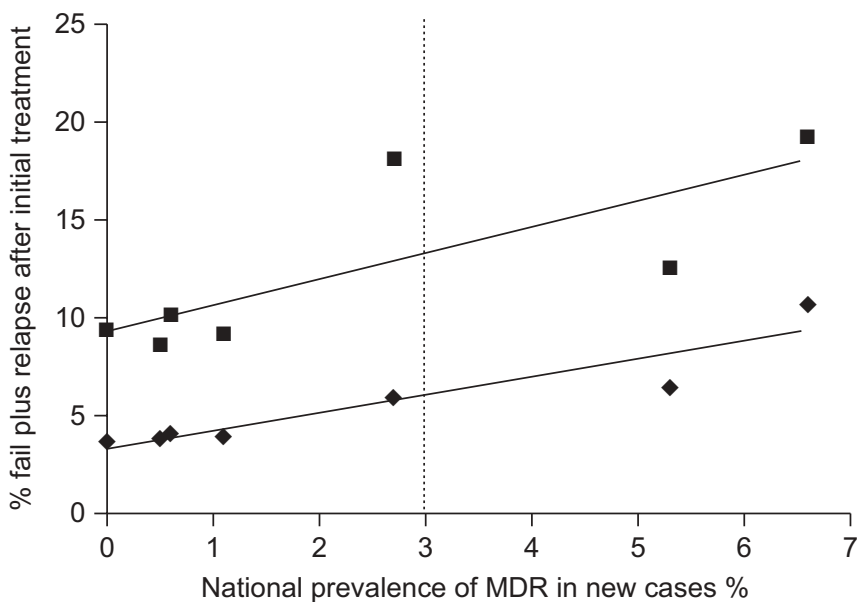

FIGURE 1. Percentage of cohort requiring retreatment (failure and relapse of initial treatment) by prevalence of multidrug-resistance (MDR) in new cases. Each point represents one of the seven countries included in the modelling. $\quad$ :-month standardised regimen; $\mathbf{\square}$ : 8-month standardised regimen. .......: the global weighted mean of prevalence of MDR in new cases $(2.9 \%)$.

due to MDR-TB. However, in the countries modelled with low prevalence of drug resistance, $>80 \%$ of deaths occurred in persons with pan-susceptible strains. The most important gain from changing from the 8-month to the 6-month initial regimen would be 4-12 fewer deaths, as seen in table 5 . This reflects that only about half the number of patients would fail or relapse following the initial 6-month regimen, thereby avoiding the risk of mortality from a second episode of active TB.

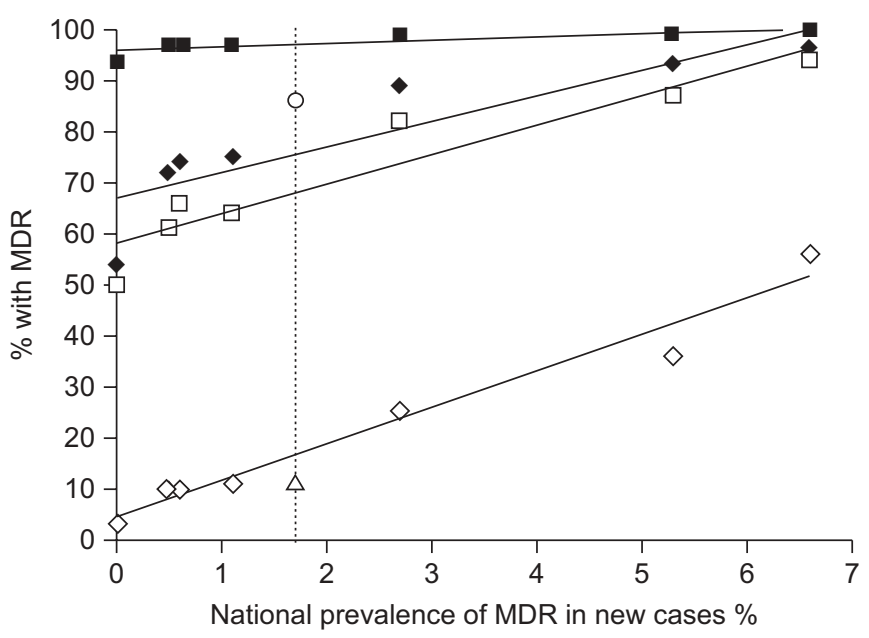

FIGURE 2. Percentage of failures and relapses with multidrug-resistance (MDR) following standardised initial treatment with 6-month regimen isoniazid, rifampin and pyrazinamide for 2 months followed by isoniazid plus rifampin for 4 months (2HRZ/4HR) and standardised retreatment, by national prevalence of MDR in new cases. Each point represents one of the seven countries included in the modelling. $\mathbf{\square}$ : failures during retreatment; $\bullet$ : relapse after retreatment; $\square$ : failures during initial therapy; $\diamond:$ relapse after initial therapy. $\cdots \cdots \cdot$. national MDR prevalence in Thailand. $\bigcirc$ : reported MDR prevalence among failures (86\%); $\triangle$ : reported MDR among relapses (11\%) [12].

\section{Sensitivity analysis}

When relapse rates were increased by $20 \%$ (to reflect the impact of default rates) results were similar to the main analysis (tables S2-S6 in the online supplementary material).

\begin{tabular}{|c|c|c|c|c|c|c|c|}
\hline \multirow[t]{3}{*}{ Country } & \multirow[t]{3}{*}{ Initial regimen } & \multicolumn{3}{|c|}{ Among failures of retreatment } & \multicolumn{3}{|c|}{ Among relapses after retreatment } \\
\hline & & \multirow{2}{*}{$\begin{array}{c}\text { Pan- } \\
\text { sensitive \% }\end{array}$} & \multicolumn{2}{|c|}{ Resistance } & \multirow{2}{*}{$\begin{array}{c}\text { Pan- } \\
\text { sensitive \% }\end{array}$} & \multicolumn{2}{|c|}{ Resistance } \\
\hline & & & Persistent $^{\#}$ & Acquired" & & Persistent ${ }^{\#}$ & Acquired \\
\hline \multirow[t]{2}{*}{ Dominican Republic } & 2HRZE/6HE & 0 & 94 & 6 & 4 & 91 & 4 \\
\hline & 2HRZE/4HR & 0 & 99 & 1 & 2 & 97 & 1 \\
\hline \multirow[t]{2}{*}{ Peru } & 2HRZE/6HE & 1 & 93 & 7 & 11 & 84 & 5 \\
\hline & 2HRZE/4HR & 0 & 99 & 1 & 6 & 93 & 1 \\
\hline \multirow[t]{2}{*}{ Vietnam } & 2HRZE/6HE & 1 & 77 & 22 & 9 & 78 & 13 \\
\hline & 2HRZE/4HR & 0 & 97 & 3 & 6 & 92 & 2 \\
\hline \multirow[t]{2}{*}{ Gambia } & 2HRZE/6HE & 3 & 86 & 10 & 41 & 54 & 5 \\
\hline & 2HRZE/4HR & 1 & 96 & 3 & 23 & 74 & 2 \\
\hline \multirow[t]{2}{*}{ Kenya } & 2HRZE/6HE & 10 & 28 & 61 & 51 & 36 & 12 \\
\hline & 2HRZE/4HR & 2 & 89 & 8 & 35 & 60 & 5 \\
\hline
\end{tabular}

Data are presented as \% and rounded to whole numbers. 2HRZE: isoniazid, rifampin, pyrazinamide and ethambutol for 2 months; $6 \mathrm{HE}$ : isoniazid plus ethambutol for 6 months; 4HR: isoniazid plus rifampin for 4 months. ${ }^{*}$ : persistent drug resistance is defined as resistance that exists at the start and remains throughout treatment; $\because$ acquired drug resistance is defined as resistance that is amplified or occurs de novo during a course of treatment. 


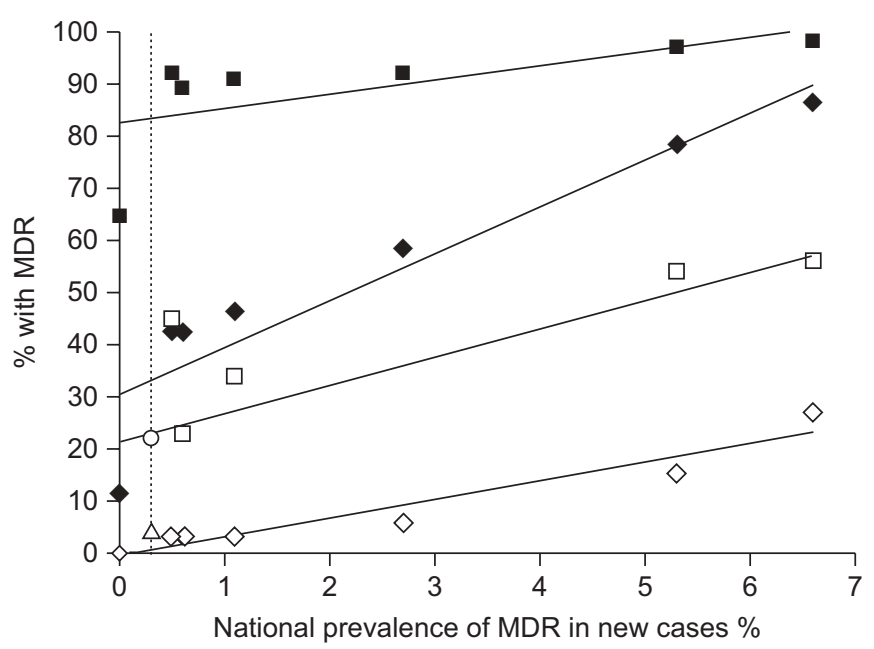

FIGURE 3. Percentage of multidrug-resistance (MDR) among failures and relapses following initial treatment with 8-month regimen isoniazid, rifampin and pyrazinamide for 2 months followed by isoniazid and ethambutol for 6 months (2HRZ/6HE) and standardised retreatment, by national prevalence of MDR in new cases. Each point represents one of the seven countries included in the modelling 口 : failures during retreatment; $\bullet$ : relapse after retreatment; $\square$ : failures during initial therapy; $\diamond$ : relapse after initial therapy. ….. national MDR prevalence in Benin $\mathrm{O}$ : reported MDR prevalence among failures (22\%); $\triangle$ : reported MDR among relapses (4\%) [15].

When the rates of acquired drug resistance during initial treatment were increased by $25 \%$, even more MDR cases were predicted to develop with use of the 8-month regimen relative to the 6-month regimen (tables S7-S11 in the online supplementary material). Even if the retreatment regimen was 25\% more efficacious, results were not substantially altered (tables S12-S16 in the online supplementary material).

\section{DISCUSSION}

The most important finding of this study is that, in all countries modelled, following initial therapy with the 6-month regimen a very high proportion of failures and relapses are predicted to be drug resistant and more than half will have MDR. Changing from the 8-month to the 6-month regimen is predicted to result in fewer deaths and half as many patients requiring retreatment, but these are much more likely to have MDR. As a result the predicted rates of failure and relapse, and proportion with drug resistance among them, will be high if the same standardised retreatment is used.

Model inputs should have been accurate as they were taken from a meta-analysis of results of 57 randomised trials conducted in many settings that included a total of 19,801 patients [4]. The algorithm developed for predicting the pattern of drug resistance in failures and relapses is simple, and the predictions were accurate in the few countries validated. This simple model (fig. 3) could be used to predict drug resistance profiles among failures and relapses in countries that do not have surveillance data for retreatment patients. While awaiting surveillance data, these predicted patterns could be used to select appropriate regimens for patients with failure or relapse.

There are several limitations to this analysis. First, model inputs were taken from randomised trials, which may underestimate the extent of acquired drug resistance in a true programme setting. This limitation was addressed in sensitivity analysis, however, and a greater acquisition of drug

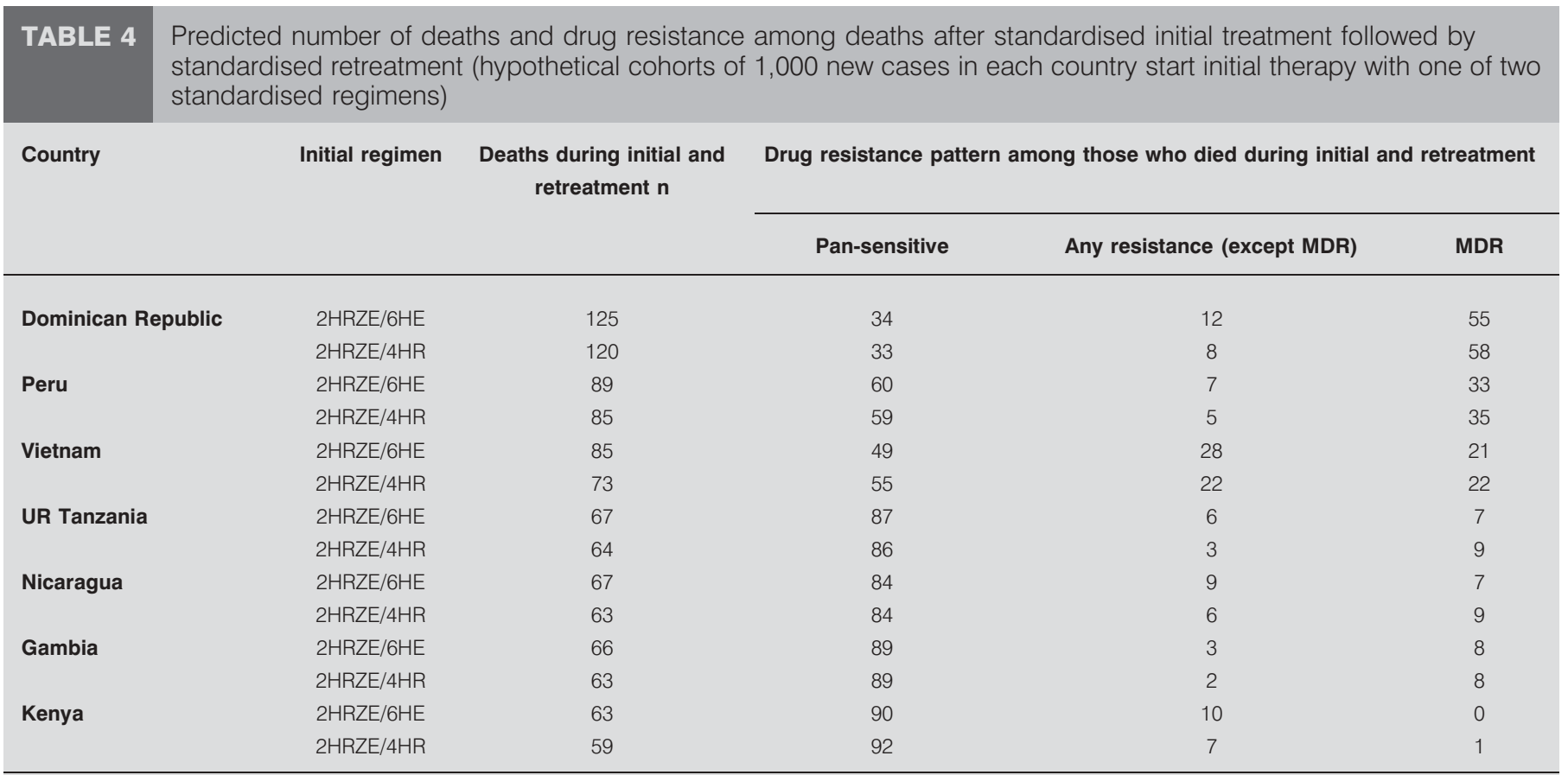

Data are presented as \% and rounded to whole numbers, unless otherwise stated. MDR: multidrug-resistance; 2HRZE: isoniazid, rifampin, pyrazinamide and ethambuto for 2 months; $6 \mathrm{HE}$ : isoniazid plus ethambutol for 6 months; $4 \mathrm{HR}$ : isoniazid plus rifampin for 4 months. 


\begin{tabular}{|c|c|c|c|c|c|}
\hline \multirow[t]{2}{*}{ Country } & \multirow[t]{2}{*}{ Initial regimen } & \multirow{2}{*}{$\begin{array}{c}\begin{array}{c}\text { Outcomes after initial } \\
\text { treatment }\end{array} \\
\begin{array}{c}\text { Patients who relapse } \\
\text { or fail } n\end{array}\end{array}$} & \multicolumn{3}{|c|}{ Outcomes after initial and retreatment ${ }^{\#}$} \\
\hline & & & Deaths & $\begin{array}{l}\text { Relapse or fail, and } \\
\text { survive with MDR-TB }\end{array}$ & $\begin{array}{l}\text { Relapse or fail, and survive with } \\
\text { any other form of DR-TB }\end{array}$ \\
\hline \multirow[t]{3}{*}{ Dominican Republic } & 2HRZE/6HE & 192 & 125 & 50 & 3 \\
\hline & 2HRZE/4HR & 106 & 120 & 51 & 0 \\
\hline & Difference $^{+}$ & 86 & 5 & -1 & 3 \\
\hline \multirow[t]{3}{*}{ Peru } & 2HRZE/6HE & 125 & 89 & 21 & 2 \\
\hline & 2HRZE/4HR & 63 & 85 & 22 & 1 \\
\hline & Difference & 62 & 4 & -1 & 1 \\
\hline \multirow{2}{*}{ UR Tanzania } & 2HRZE/4HR & 39 & 63 & 6 & 0 \\
\hline & Difference & 53 & 4 & -1 & 1 \\
\hline \multirow[t]{3}{*}{ Nicaragua } & 2HRZE/6HE & 101 & 67 & 4 & 1 \\
\hline & 2HRZE/4HR & 40 & 63 & 5 & 0 \\
\hline & Difference & 61 & 4 & -1 & 1 \\
\hline \multirow[t]{3}{*}{ Gambia } & 2HRZE/6HE & 86 & 66 & 4 & 1 \\
\hline & 2HRZE/4HR & 38 & 63 & 5 & 0 \\
\hline & Difference & 48 & 3 & -1 & 1 \\
\hline \multirow[t]{3}{*}{ Kenya } & 2HRZE/6HE & 94 & 63 & 1 & 1 \\
\hline & 2HRZE/4HR & 36 & 59 & 2 & 0 \\
\hline & Difference & 58 & 4 & -1 & 1 \\
\hline
\end{tabular}

Data are rounded to whole numbers. MDR: multidrug-resistance; TB: tuberculosis; DR: drug resistance; 2HRZE: isoniazid, rifampin, pyrazinamide and ethambutol for 2 months; $6 \mathrm{HE}$; isoniazid plus ethambutol for 6 months; 4HR: isoniazid plus rifampin for 4 months ${ }^{\#}$ : total number from original hypothetical cohort of 1,000 new cases;

$\because$ : not MDR-TB; ${ }^{+}$: difference is positive if outcome more frequent with 8 -month regimen, and negative if outcome more frequent with 6 -month regimen.

resistance during initial treatment only led to increased MDR, especially with the 8-month regimen. Secondly, the risk of acquiring drug resistance in HIV-positive TB patients was assumed to be the same as in HIV-negative ones, because there is limited information on this point [16]. Again, the potential impact of increased acquired drug resistance was addressed in sensitivity analyses, but predictions may not be accurate for high HIV burden settings. Thirdly, default was not included in the primary analysis; as shown in the sensitivity analysis, higher default rates would result in more persons requiring retreatment and more deaths. Default rates are higher in countries using the 8-month regimen [10]. Therefore, assuming that a switch to a shorter regimen would in turn reduce the default rate and improve outcomes, the decision not to include default would tend to underestimate the advantages of switching from the 8-month to the 6-month regimen. Fourthly, we assumed that all failures and relapses would be detected and treated. This would result in an underestimate of mortality among failures and relapses, since it is unlikely they would all be detected under programme conditions. Finally, the model also did not estimate transmission from failures and relapses resulting in secondary cases. These last two limitations would also underestimate the advantages of changing from the 8-month regimen.
An obvious limitation is the lack of specific input data for treatment outcomes in patients with various forms of drug resistance receiving the standardised retreatment regimen. However, in a recent systematic review we could find no randomised trials, and only three reports of outcomes with the standardised retreatment regimen in three small cohorts, each with 30-40 patients with isoniazid mono-resistance [6]. Hence, there are simply no published data available on which to base predictions.

This analysis has two major implications. First, we predict that changing from the standardised 8-month regimen to the 6month regimen to treat new cases will result in fewer deaths, and substantially fewer patients who fail or relapse and therefore require retreatment. Differences will be greater in countries with higher levels of initial drug resistance. These findings provide strong support for the recommendation to switch from the 8-month to the 6-month initial regimen.

However, the most important implication is the need for a better retreatment strategy following initial therapy with the 6month regimen. In almost all countries, more than half of all closely supervised patients who fail this initial regimen are predicted to have MDR-TB, while in countries with high prevalence of initial drug resistance more than half of relapses 
will have MDR. Because of this, we predict that the current retreatment regimen will have low efficacy in many settings.

The current retreatment strategy was designed empirically over 25 yrs ago [7], and is now used to treat $\geqslant 1$ million patients annually. Given its origins, current widespread use, and the recent change in WHO recommendations [5], our findings support calls [8,9] for several changes: 1) improved drug resistance surveillance linked to detailed clinical histories; this could be implemented rapidly to provide information to guide design of appropriate regimens in different settings; 2) access to drug sensitivity testing for all retreatment patients; and 3) strengthened retreatment regimens. There can be no doubt that use of standardised regimens has enhanced access to treatment for patients in many settings. However, the identification of standardised regimens that are the most efficacious and least toxic for patients requiring retreatment in all countries will take a concerted international effort. Surveillance studies and a series of randomised trials will be needed to adequately evaluate the best options for retreatment, to resolve what is now the Achilles heel of the directly observed treatment, short course strategy.

\section{SUPPORT STATEMENT}

Funding for this modelling study was provided, in part, from the World Health Organization. Salary support was provided by the Canadian Institutes of Health Research for M. Pai and O. Oxlade (Respiratory Epidemiology and Clinical Research Unit, Montreal Chest Institute, McGill University, and Dept of Epidemiology, Biostatistics and Occupational Health, McGill University, Montreal, Canada), and the Fonds de la recherche en santé du Québec for D. Menzies (Respiratory Epidemiology and Clinical Research Unit, Montreal Chest Institute, McGill University) and A. Benedetti (Respiratory Epidemiology and Clinical Research Unit, Montreal Chest Institute, McGill University, and Dept of Epidemiology, Biostatistics and Occupational Health, McGill University). None of these agencies had any direct role in the conduct of the study, or the decision to submit the manuscript for publication.

\section{STATEMENT OF INTEREST}

None declared.

\section{ACKNOWLEDGEMENTS}

The authors would like to thank M. Grzemska (Division of Tuberculosis, WHO, Geneva, Switzerland) for assistance throughout this project.

\section{REFERENCES}

1 World Health Organization. Treatment of Tuberculosis: Guidelines for National Programmes WHO/CDS/TB/2008 (Revised June 2008). 3rd Edn. Geneva, World Health Organization, 2008; p. 313.

2 Enarson DA, Rieder HL, Arnadottir T, et al. Tuberculosis guide for low income countries. 3rd Edn. Paris, International Union Against Tuberculosis and Lung Disease, 2000.
3 World Health Organization. Global Tuberculosis Control Surveillance, Planning, Financing: 2008 Report No.: (WHO/ HTM/TB/2006.362). Geneva, World Health Organization, 2008.

4 Menzies D, Benedetti A, Paydar A, et al. Effect of duration and intermittency of rifampin on tuberculosis treatment outcomes: a systematic review and meta-analysis. PLOS Med 2009; 6: e1000146.

5 World Health Organization. Treatment of Tuberculosis Guidelines: 4th Edn Report No.: WHO/HTM/TB/2009.420. Geneva, World Health Organization, 2010.

6 Menzies D, Benedetti A, Paydar A, et al. Standardized treatment of active tuberculosis in patients with previous treatment and/or with mono-resistance to isoniazid: a systematic review and metaanalysis. PLOS Med 2009; 6: e1000150.

7 Rouillon A. The mutual assistance programme of the IUATLD. Development, contribution and significance. Bull Int Union Tubec Lung Dis 1991; 66: 159-172.

8 Espinal MA Time to abandon the standard retreatment regimen with first-line drugs for failures of standard treatment, Int J Tuberc Lung Dis 2003; 7: 607-608.

9 Espinal M, Raviglione MC. From threat to reality: the real face of multidrug-resistant tuberculosis. Am J Resp Crit Care Med 2008; 178: 216-217.

10 Mak A, Thomas A, Granado M, et al. Influence of multidrug resistance on tuberculosis treatment outcomes with standardized regimens. Am J Respir Crit Care Med 2008; 178: 306-312. 2008.

11 Quy HTW, Lan NTN, Borgdorff MW, et al. Drug resistance among failure and relapse cases of tuberculosis: is the standard retreatment regimen adequate? Int J Tuberc Lung Dis 2003; 7: 631-636.

12 Yoshiyama T, Yanai H, Rhiengtong D, et al. Development of acquired drug resistance in recurrent tuberculosis patients with various previous treatment outcomes. Int J Tuberc Lung Dis 2004; 8: 31-38.

13 World Health Organization. Anti-tuberculosis drug resistance in the world report number 4 Report No.: WHO/HTM/TB/2008. Geneva, World Health Organization, 2008. Available from: whqlibdoc.who.int/publications/2008; 2008.

14 Becerra MC, Freeman J, Bayona J, et al. Using treatment failure under effective directly observed short-course chemotherapy programs to identify patients with multidrug-resistant tuberculosis. Int J Tuberc Lung Dis 2000; 4: 108-114.

15 Trébucq A, Anagonou S, Gninafon M, et al. Prevalence of primary and acquired resistance of Mycobacterium tuberculosis to antituberculosis drugs in Benin after 12 years of short-course chemotherapy. Int J Tuber Lung Dis 1999; 3: 466-470.

16 Khan FA, Minion J, Pai M, et al. Treatment of active tuberculosis in HIV co-infected patients: a systematic review and meta-analysis. Clin Infect Dis 2010; 50: 1288-1299.

17 Vernon A, Burman W, Benator D, et al. Acquired rifamycin monoresistance in patients with HIV-related tuberculosis treated with once-weekly rifapentine and isoniazid. Tuberculosis Trials Consortium. Lancet 1999; 353: 1843-1847.

18 Lew $\mathrm{W}$, Oxlade $\mathrm{O}$, Pai $\mathrm{M}$, et al. Initial drug resistance and tuberculosis treatment outcomes: systematic review and metaanalysis. Ann Intern Med 2008; 149: 123-134.

19 Rieder HL. Epidemiologic Basis of Tuberculosis Control. 1st Edn. Paris, International Union Against Tuberculosis and Lung Disease, 1999; pp. 1-162. 